\title{
Response to Elwood, M. et al., Comment on: Maternal Exposure to Domestic Hair Cosmetics and Occupational Endocrine Disruptors Is Associated with a Higher Risk of Hypospadias in the Offspring. Int. J. Environ. Res. Public Health 2017, 14, 27
}

\author{
Elodie Haraux ${ }^{1, *}$ (D), Karine Braun ${ }^{2}$, Philippe Buisson ${ }^{1}$, Erwan Stéphan-Blanchard ${ }^{3}$, \\ Camille Devauchelle ${ }^{4}$, Jannick Ricard ${ }^{1}$, Bernard Boudailliez ${ }^{2}$, Pierre Tourneux ${ }^{5}$ (D), \\ Richard Gouron ${ }^{1}$ and Karen Chardon ${ }^{3}$ \\ 1 Department of Paediatric Surgery, Amiens University Hospital, 80054 Amiens, France; \\ buisson.philippe@chu-amiens.fr (P.B.); ricard.jannick@chu-amiens.fr (J.R.); \\ gouron.richard@chu-amiens.fr (R.G.) \\ 2 Department of Paediatrics, Amiens University Hospital, 80054 Amiens, France; \\ braun.karine@chu-amiens.fr (K.B.); boudailliez.bernard@chu-amiens.fr (B.B.) \\ 3 PériTox-INERIS Laboratory, Jules Verne University of Picardy, 80054 Amiens, France; \\ erwan.stephan@u-picardie.fr (E.S.-B.); karen.chardon@u-picardie.fr (K.C.) \\ 4 Department of Paediatrics, Creil Hospital, 60100 Creil, France; camille.devauchelle@ch-creil.fr \\ 5 Department of Paediatric Intensive Care Unit, Amiens University Hospital, 80054 Amiens, France; \\ tourneux.pierre@chu-amiens.fr \\ * Correspondence: haraux.elodie@chu-amiens.fr; Tel.: +33-322087563
}

Received: 24 May 2017; Accepted: 4 August 2017; Published: 15 September 2017

Dear Editor,

Thank you for inviting us to reply to a "Comment" paper to our published paper "Maternal Exposure to Domestic Hair Cosmetics and Occupational Endocrine Disruptors Is Associated with a Higher Risk of Hypospadias in the Offspring" (Authors: Elodie Haraux, Karine Braun, Philippe Buisson, Erwan Stéphan-Blanchard, Jannick Ricard, Camille Devauchelle, Bernard Boudailliez, Pierre Tourneux, Richard Gouron, Karen Chardon).

We thank the comment's authors for highlighting some inconsistencies in the published results [1]. After verification, we acknowledge that incorrect data were reported in Table 4, and could have led to misinterpretation of our results. A corrected version of this table is provided below. Importantly, these changes do not modify the significance of the results or their related conclusions. Also, there is now no significant difference for missing data on use of hair cosmetics between cases $(17.5 \%)$ and controls $(18.5 \%)$. Our study is the first to report an impact of the use of domestic hair cosmetics (not only EDCs occupational exposure per se) on the risk of hypospadias.

As pointed out by the comment's authors, the participation rate was not recorded in our study but this limitation was clearly indicated in the 'Discussion' section of the manuscript.

As questioned by the comment's authors, the strong increase between the univariate and multivariate OR for EDCs occupational exposure was mainly due to paternal weight. This specific point was discussed in the 'Parental risk factors and medication' section of the discussion.

Concerning occupational maternal exposure, we first calculated only for women who worked, and secondly for all women. So when women were housewives, we considered no occupational exposure. These results were presented in Table 4. We also acknowledge that incorrect data were reported about "working during pregnancy" (23 instead of 19 women were housewives in the 
Hypospadias group vs. 76 and not 65 in the control group). OR was higher when calculated for "working women" (univariate 3.6 (1.4-9.3) and multivariate 9.6 (1.41-66.09)) than for all the women (univariate 3.1 (1.3-7.6) and 5.1 (1.1-23.06)). It was a choice to present the first result but in both cases, the association between occupational exposure and hypospadias persisted.

The authors would like to apologize for any inconvenience caused to the readers.

Elodie Haraux and co-authors.

Table 4. Univariate analysis of the association between pollutant exposures during the first trimester of pregnancy and the incidence of hypospadias.

\begin{tabular}{|c|c|c|c|c|}
\hline & & Cases $(n=57)$ & Controls $(n=162)$ & $p$-Value, OR $(95 \% \mathrm{CI})$ \\
\hline \multicolumn{5}{|c|}{ COSMETICS } \\
\hline \multirow{2}{*}{ Hair cosmetics ( $n$ ) } & Yes & 25 & 51 & 0.07 \\
\hline & No & 22 & 81 & $1.9(0.9-3.5)$ \\
\hline \multirow{2}{*}{-Hairspray $(n)$} & Yes & 16 & 37 & 0.4 \\
\hline & No & 31 & 95 & $1.3(0.6-2.7)$ \\
\hline \multirow{2}{*}{-Colouring shampoo $(n)$} & Yes & 13 & 25 & 0.2 \\
\hline & No & 34 & 105 & $1.6(0.7-3.5)$ \\
\hline \multicolumn{5}{|c|}{ CHEMICALS } \\
\hline \multirow{2}{*}{ Chemicals $(n)$} & Yes & 41 & 120 & 0.38 \\
\hline & No & 6 & 11 & $0.6(0.2-1.8)$ \\
\hline \multirow{2}{*}{-Paint/solvents/gasoline $(n)$} & Yes & 5 & 23 & 0.3 \\
\hline & No & 42 & 108 & $0.6(0.2-1.6)$ \\
\hline \multirow{2}{*}{-Ink $(n)$} & Yes & 3 & 6 & 0.6 \\
\hline & No & 44 & 124 & NA \\
\hline \multirow{2}{*}{-Glue (n) } & Yes & 5 & 13 & 0.90 \\
\hline & No & 42 & 118 & $1.1(0.4-3.2)$ \\
\hline \multirow{2}{*}{-Household products ( $n$ ) } & Yes & 41 & 120 & 0.6 \\
\hline & No & 5 & 11 & $0.8(0.2-2.3)$ \\
\hline \multirow{2}{*}{ Human antiparasitic $(n)$} & Yes & 13 & 57 & 0.10 \\
\hline & No & 38 & 93 & $0.56(0.3-1.1)$ \\
\hline \multicolumn{5}{|c|}{ ENVIRONMENTAL FACTORS } \\
\hline \multirow{2}{*}{ Living $<1 \mathrm{~km}$ from a field } & Yes & 33 & 76 & 0.07 \\
\hline & No & 14 & 61 & $1.9(0.9-3.8)$ \\
\hline \multirow{2}{*}{ Living $<1 \mathrm{~km}$ from a factory } & Yes & 12 & 43 & 0.3 \\
\hline & No & 31 & 78 & $0.7(0.3-1.5)$ \\
\hline \multirow{2}{*}{ Garden $(n)$} & Yes & 40 & 99 & $0.04 *$ \\
\hline & No & 8 & 46 & $2.3(1.0-5.3)$ \\
\hline \multirow{2}{*}{ Pets $(n)$} & Yes & 38 & 85 & $0.02 *$ \\
\hline & No & 13 & 65 & $2.2(1.1-4.5)$ \\
\hline \multirow{2}{*}{ Veterinary insecticides ( $n$ ) } & Yes & 37 & 84 & $0.04 *$ \\
\hline & No & 14 & 65 & $2.0(1.02-4.1)$ \\
\hline \multicolumn{5}{|c|}{ OCCUPATIONAL FACTORS } \\
\hline \multirow{2}{*}{ Working during pregnancy $(n)$} & Yes & 32 & 86 & 0.6 \\
\hline & No & 23 & 76 & $1.2(0.7-2.3)$ \\
\hline \multirow{2}{*}{ Occupational exposure to EDCs (JEM) ( $n$ ) } & Yes & 11 & 12 & $0.007 * *$ \\
\hline & No & 44 & 150 & $3.6(1.4-9.3)$ \\
\hline
\end{tabular}

Reference: ${ }^{*} p<0.05 ;{ }^{* *} p<0.01$. 
Author Contributions: Elodie Haraux and Karen Chardon and Karine Braun conceptualized and designed the study. Elodie Haraux and Karen Chardon drafted the initial manuscript, and approved the final manuscript as submitted. Pierre Tourneux, Philippe Buisson, Camille Devauchelle and Karine Braun designed the data collection instruments, and coordinated and supervised data collection. Richard Gouron, Bernard Boudailliez, Erwan Stéphan-Blanchard and Jannick Ricard revised the manuscript, and approved the final manuscript as submitted. Elodie Haraux, Erwan Stéphan-Blanchard and Karen Chardon wrote the reply.

Conflicts of Interest: The authors have no conflicts of interest relevant to this article to disclose.

\section{References}

1. Haraux, E.; Braun, K.; Buisson, P.; Stéphan-Blanchard, E.; Devauchelle, C.; Ricard, J.; Boudailliez, B.; Tourneux, P.; Gouron, R.; Chardon, K. Maternal Exposure to Domestic Hair Cosmetics and Occupational Endocrine Disruptors Is Associated with a Higher Risk of Hypospadias in the Offspring. Int. J. Environ. Res. Public Health 2016, 14, 27. [CrossRef] [PubMed] 